\title{
Project Seattler For RENEWABLE ELECTRICITY
}

\author{
Rugescu, R.D.; TACHE, F.; ChiciudeAn, T.G.; TOMA A.C.; \\ SLAVU, B. \& GALAN, V.
}

Abstract: The objective of the research described in this paper was to develop numerical simulations and to compare the results with laboratory experiments in new, promising zones of aeroacoustics, considered fruitful for the environmental technologies, particularly related to aerodynamic noise suppression. When noise measurements are not being conducted, the cold air turbine embedded in the tower can be used as an efficient, eco-friendly source of energy.

Named SEATTLER from Solar Energy Actuator for Tall Tower Low-cost Electricity Research, this moderately tall tower creates a considerable air acceleration and allows the aerodynamic noise to be definitely perceived. Numeric simulations applied on this architecture reveal astonishing air velocities well over $100 \mathrm{~m} / \mathrm{s}$ in the test chamber area.

Key words: gravitational draught, infra-turbulence wind tunnel, solar energy, cold air turbine
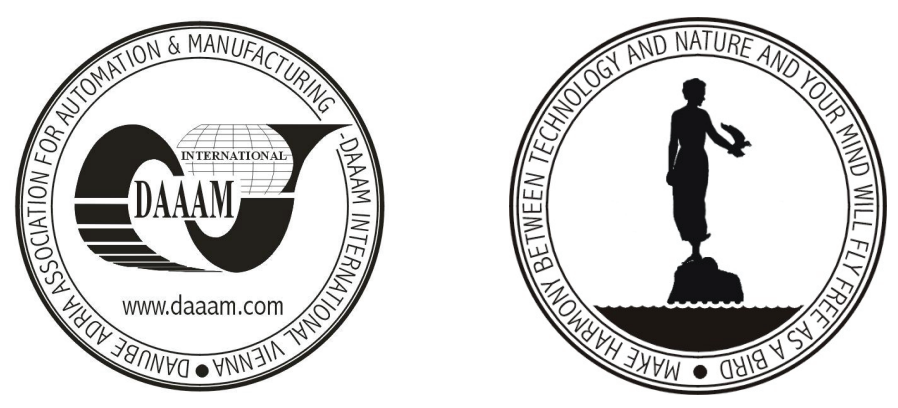

Authors data: Dr. Sc. Rugescu R.[adu] D.[an] \& Eng. MSc student Researcher Tache F.[lorin], Eng. MSc student Slavu B.[ernard], Eng. MSc student Galan V.[iorel], Eng. PhD student Chiciudean T.[eodor] G.[elu], Eng. PhD \& MSc stud. Toma A.[dina] C.[ristina], University "POLITEHNICA" of Bucharest, Romania, rugescu@yahoo.com, flotasoft@yahoo.com,_slavu_bernard@yahoo.com, teodorgelu@yahoo.com, tomaadinacristina@yahoo.com

This Publication has to be referred as: Rugescu, R.D.; Tache, F.; Chiciudean, T. G.; Toma, A.C.; Slavu, B. \& Galan, V. (2006). Project Seattler for Renewable Electricity, Chapter 42 in DAAAM International Scientific Book 2006, B. Katalinic (Ed.), Published by DAAAM International, ISBN 3-901509-47-X, ISSN 1726-9687, Vienna, Austria

DOI: $10.2507 /$ daaam.scibook.2006.42 